\title{
Preferencias y hábitos de lectura en estudiantes universitarios
}

\section{Reading preferences and habits in college students}

Recepción del artículo: 04-04-2021 | Aceptación del artículo: 10-12-2021

Edward Faustino Loayza-Maturrano

edwloma@lamolina.edu.pe

iD https://orcid.org/0000-0002-1359-8414

Universidad Nacional Agraria La Molina, Perú

\section{Resumen}

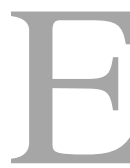

l propósito de la investigación es determinar las preferencias de lectura y la existencia del hábito lector en estudiantes universitarios de los últimos semestres de estudios de formación académica. El diseño del estudio es cuantitativo de tipo descriptivohermenéutico. La recogida de información se efectuó a través de una encuesta de preferencias personales y actitudes generales hacia la lectura validada en el estudio de Ögeyik \& Akyay. La encuesta de 40 ítems se aplicó a 54 estudiantes universitarios de los últimos semestres académicos de una universidad pública. Los resultados muestran que los estudiantes universitarios tienen actitudes positivas hacia la lectura y se encuentran totalmente involucrados en actividades lectoras. Se concluye que los estudiantes universitarios tienen hábitos lectores e intereses de lectura, porque evidencian ser lectores conscientes: investigan sobre los libros antes de comprarlos y muestran la tendencia general a elegir sus textos según la necesidad de la información que les interesa.

Palabras Clave: Diagnóstico de lectura; interés por la lectura; actitudes hacia la lectura; hábito lector; estudiantes universitarios.
Loayza-Maturrano, E. F. (2022). Preferencias y hábitos de lectura en estudiantes universitarios. Revista ConCiencia EPG,

$7(1), 36-51$. https://doi.org/10.32654/CONCIENCIAEP

G.7-1.3

Para referenciar este artículo: 
Abstract

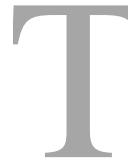

he purpose of the research is to determine the reading preferences and the existence of the reading habit in university students in the last semesters of academic training. The study design is quantitative and descriptive-hermeneutical. Information was collected through a survey of personal preferences and general attitudes towards reading validated in the Ögeyik \& Akyay study. The 40-item survey was applied to 54 university students from the last academic semesters of a public university. The results show that university students have positive attitudes towards reading and are fully involved in reading activities. It is concluded that university students have reading habits and reading interests, because they show that they are conscious readers: they investigate books before buying them and show the general tendency to choose their texts according to the need for the information that interests them.

Key Words: Reading diagnostics; reading interests; reading attitudes; reading habits; college students.

\section{Introducción}

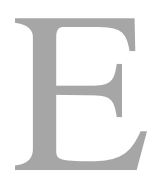

n las universidades públicas del Perú, en particular de los estudiantes que se forman en carreras como las de las ingenierías y las ciencias naturales han reducido sustancialmente las horas lectivas destinadas a curso de formación general y en especial en el ámbito de las humanidades. Las estructuras curriculares se han orientado hacia un enfoque principal centrado en la hiperespecialización (Delgado, 2020). Es por este hecho que se hace necesario constatar las preferencias de lectura y la existencia del hábito lector en estudiantes universitarios que han sido formados con la currícula anterior; en la que se otorgaba mayor importancia a los cursos de formación general como aquellos relacionados con el desarrollo de la habilidad de la lectura: los cursos del área de lenguaje (Casiraghi, \& Soares, 2021). Los nuevos currículos ponen límites en la cantidad de créditos académicos, lo cual podría provocar en los futuros profesionales debilidades en la formación de capacidades específicas relacionadas con la lectura (Sotelo y Espíritu, 2020).

La lectura siempre se ha considerado importante ya que proporciona información comprensible para que los estudiantes adquieran un nuevo conocimiento. Además, la efectividad en la lectura inevitablemente se relaciona con el aprendizaje y la investigación para fines específicos. Por lo tanto, la reducción de carga lectiva a cursos de formación general, en especial de los cursos del área de lenguaje, supone también limitar la adquisición de nuevos conocimientos, contextos y vocabulario específico, pues se debilita el desarrollo de las habilidades cognitivas relacionadas con la lectura. A los universitarios leer menos, desconocer de los métodos, técnicas y estrategias de lectura podría impedirles comprender el texto académico e incluso desarrollar la comunicación (Flores, 2016; Bao, 2019). 
La lectura juega un papel importante en el enriquecimiento y la ampliación del conocimiento y la comprensión de los lectores en ciertos campos, sin importar que los lectores lean textos particulares en su lengua materna o en una segunda lengua como el inglés o portugués. En este sentido, la lectura se convierte en una forma efectiva para que los estudiantes universitarios adquieran conocimientos. Por lo tanto, la lectura se considera generalmente como un medio eficaz para conseguir nueva información y conocimiento; ya que es un hábito que se adquiere a largo plazo, el cual comienza en edades muy tempranas, y es el medio principal para el aprendizaje y el conocimiento. Por lo tanto, la lectura es un proceso crucial de desarrollo individual de capacidades para comprender la información predominante del mundo (Ögeyik \& Akyay, 2009; Domínguez, et al., 2015).

Tener el hábito de leer no es sencillo para la mayoría de los lectores. Un hábito es producto del aprendizaje y de una actitud positiva; por lo que los hábitos de lectura son actividades de lectura que se realizan de forma rutinaria, consciente e intencionada (Salazar y Ponce, 1999; Guevara, 2017). Este punto de vista muestra que los lectores deben ensayar su lectura para convertirla en un buen hábito. Por tanto, para incentivar el hábito lector de los estudiantes universitarios, se recomienda fomentar el deseo de leer y los textos deben ser variados y en función de los fines para los que los estudiantes quieren leer en cada circunstancia: libros, revistas, periódicos, ficción, no ficción; textos que informan, textos que entretienen, textos de divulgación general, especializados, ligeros o serios (De la Puente, 2015). Por lo tanto, al hacerlo de forma continua, los estudiantes se activan y adquieren el hábito de lectura.

Los hábitos de lectura son esenciales para las más jóvenes generaciones, especialmente estudiantes universitarios porque ellos competirán en los mercados globales del futuro. En tal sentido, no es sorprendente que muchos países dan mucha atención a los hábitos de lectura de su población, especialmente en edades tempranas. La importancia del hábito de la lectura en edades tempranas es equiparable a los niños con las habilidades de aprender a leer y leer para aprender a mejorar su capacidad general para el aprendizaje permanente y el desarrollo integral de la persona (Salvador y Agustín, 2015). Por lo tanto, los hábitos de lectura no deben subestimarse nunca para concientizar a las personas de que la lectura es una necesidad como parte de sus vidas (Muñoz et al., 2016).

En el contexto educativo actual existen muchos factores que influyen en si los estudiantes tienen altos niveles de motivación hacia la lectura, o si tienen o no el hábito lector. La motivación lectora de los estudiantes está influenciada por un buen ambiente para leer, la lectura en voz alta de los padres en la niñez, las obras literarias estupendas y los buenos hábitos de lectura (Jiménez-Pérez, 2018). En este sentido, para tener un buen hábito lector los estudiantes universitarios requieren tener circunstancias favorables y propicias ya sea en sus entornos cercanos 0 en sus preferencias lectoras. De este modo, en el progreso continuo de su proceso formativo 
los universitarios necesitan mejorar, definitivamente, sus hábitos de lectura para lograr la mejora individual durante el proceso de su profesionalización (GarcíaDelgado, 2011).

Contrariamente, con el avance de la tecnología y los medios virtualizados, se puede decir que los estudiantes tienen menos tiempo para leer o incluso no tienen tiempo para dicha actividad. Los estudiantes en vez de aprovechar la facilidad en el acceso a la información para el estudio, el aprendizaje o la investigación, se ocupan de actividades recreativas (Loayza-Maturrano, 2021 a). La mayoría de los universitarios pueden pasar sus momentos de ocio viendo televisión, escuchando música, jugando juegos electrónicos o participando en redes sociales como Facebook, Twitter, Instagram (Magadán-Díaz \& Rivas-García, 2019; Elche y Yubero, 2019). En consecuencia, los estudiantes dedican más tiempo a esas cosas que a leer, por lo que las preferencias sobre textos de lectura se van restringiendo mucho. Sin embargo, dado que a los estudiantes universitarios se les considera como profesionales del futuro, y se supone que éstos tienen más tiempo para leer que otros jóvenes de su misma edad, quienes no se encuentran en la educación terciaria; se hace necesario y justificable el investigar acerca de los hábitos y preferencias de lectura de los futuros profesionales con el propósito de observar si los universitarios están influenciados por las condiciones indicadas, o si muestran preferencias de lectura variadas debido al avance de la tecnología y los medios (Álvarez y Fabregat, 2017; Fraga, 2019).
En cuanto a las investigaciones anteriores en este campo de estudio, es posible señalar que se han realizado muchas investigaciones que abordan los hábitos de lectura de los estudiantes en distintos niveles de lectores, teniendo en cuenta diferencias de edades, dominio del lenguaje, nivel de instrucción, etc. (Ögeyik \& Akyay, 2009; García-Delgado, 2011; Travieso et al, 2013; De la Puente, 2015; Jiménez-Pérez, 2018). En este contexto, los hábitos de lectura deben ser considerados importantes en cada una de las diversas situaciones. De hecho, también es necesario prestar mucha atención a los factores que influyen en los hábitos de lectura, ya que pueden bien reorientarlos o bien determinarlos. Además, las preferencias y los intereses de los lectores también pueden influir en las actitudes hacia la lectura (Kaya, Kaya, \& Bindak, 2020).

En relación con los hábitos de lectura de los estudiantes universitarios y sus preferencias de lectura, son muchas las nociones que ofrecen muchos investigadores. Un buen hábito de lectura es necesario para un crecimiento intelectual saludable y juega un papel muy importante para permitir que una persona logre la eficiencia práctica (De la Puente, 2015). Por consiguiente, la autora explica que los intereses de un individuo están determinados en gran medida por la cantidad que leerá y la intensidad con la que llevará a cabo su actividad lectora. En su estudio, revela que los hábitos de lectura están asociados con el género, la edad, la formación académica, el rendimiento académico y el crecimiento profesional de los estudiantes. Las principales concomitancias de estos factores con el 
hábito lector muestran que a mayor edad mayor hábito de lectura, que mayor escolaridad supone una mayor frecuencia lectora y que la prevalencia se inclina hacia el género femenino. Además, Parodi, et al. (2019) define que un buen hábito de lectura es una herramienta importante para el desarrollo de personalidades y capacidades mentales. Examina los hábitos de lectura de los estudiantes chilenos en la era actual de la tecnología y la virtualidad. Los resultados de la investigación revelan que los participantes prefieren los medios electrónicos cuando leen por placer, pero prefieren los medios impresos para aprobar las evaluaciones de curso.

Según lo observado en los hallazgos anteriores, se puede inferir que los hábitos de lectura en las personas pueden variar en función de varios factores. En sentido general, Alasdair Gleed (2013) sugiere que la elección de textos de lectura también puede depender de esos factores. Por ejemplo, en entornos educativos, a los alumnos como lectores se les suele dirigir a leer como una tarea obligatoria y los materiales para leer se eligen como requisito del proceso educativo. Por tanto, la presente investigación intenta indagar en las siguientes cuestiones: 1) ¿Cómo son los hábitos de lectura de los estudiantes universitarios en los últimos semestres de formación académico-profesional? 2) ¿Qué tipo de libros o textos en general son los preferidos para leer por los universitarios que cursan los últimos semestres académicos? En consecuencia, el objetivo de este estudio es determinar las preferencias de lectura y el hábito lector en los estudiantes universitarios de los últimos semestres de formación profesional en la educación universitaria pública. A partir de este estudio, los hallazgos proporcionarán información sobre las actitudes generales hacia la lectura, así como de las percepciones, preferencias, intereses y efectos de la lectura.

\section{Método}

Diseño: El estudio es de diseño transversal no experimental y de tipo descriptivo porque se aplica un cuestionario cuyos resultados permiten realizar un análisis estadístico descriptivo con el propósito de referir a partir de la frecuencia y los valores porcentuales las características de los estudiantes universitarios respecto de sus intereses de lectura y de su hábito lector (Loayza, 2020). Seguidamente, se procede con un análisis de los resultados para interpretar la situación en que se encuentran los universitarios sobre sus actitudes generales hacia la lectura y sus preferencias de lectura.

Participantes: La selección de los participantes se efectuó considerando los criterios de inclusión y exclusión siguientes: $1^{\circ}$. Que fueran estudiantes de los últimos semestres (del séptimo al décimo semestre) de estudio de una universidad pública y que pertenecieran al mismo grupo-aula en cada caso, para aplicar el cuestionario y $2^{\circ}$. Que los participantes fueran mixtos (hombres y mujeres) dentro de cada grupo. Las encuestas fueron aplicadas a 54 estudiantes universitarios de la Universidad Nacional Agraria La Molina (UNALM) distribuidos en tres grupos-aulas; 
todos ellos en calidad de participantes en el curso de Redacción Técnica. La muestra estadística fue no probabilística, de tipo muestreo por conveniencia en función del acceso y factibilidad de la población de parte del investigador.

Instrumento. Se empleó una encuesta de intereses personales $y$ actitudes generales hacia la lectura elaborada por los investigadores Ögeyik \& Akyay (2009), la cual fue validada y aplicada a estudiantes universitarios en Turquía. La encuesta consta de 40 ítems que fueron diseñados para evaluar las actitudes generales hacia la lectura y los intereses de lectura de los estudiantes universitarios. Cada ítem posee una valoración dicotómica: 1) sí, sí es cierto y 2) no, no es verdad. Los estudiantes completaron el cuestionario durante 20 minutos. De los 40 ítems, 10 ítems buscan actitudes generales hacia la lectura o hábitos de lectura, 13 ítems pertenecen a las preferencias de lectura de los participantes, 10 ítems buscan los efectos de la lectura en el desempeño de los estudiantes y 7 ítems investigan acerca las opiniones negativas sobre la lectura. El orden de las expresiones positivas y negativas se ordenó cuidadosamente para no afectar la objetividad de los sujetos.

Procedimiento. La manera de aplicación de la encuesta fue colectiva en tres grupos-aulas participantes del curso de Redacción Técnica. Los encuestados dieron su consentimiento informado antes de la resolución del instrumento. Se aplicó en el periodo de educación a distancia en el año 2020 y 2021 a través de la plataforma Zoom Meeting y empleando un cuestionario preparado previamente en la plataforma
Google Forms que fue facilitado por el docente del curso a los estudiantes a través de un enlace en el chat de la sesión sincrónica en la plataforma Zoom. La aplicación duró veinte minutos. Durante el proceso de aplicación del instrumento se complementó las instrucciones escritas de la encuesta con instrucciones verbales de parte del aplicador, expresando la naturaleza del estudio, la importancia de la honestidad en las respuestas, el consentimiento informado y el carácter anónimo del instrumento. Posteriormente, el análisis de la información se realizó en dos momentos: primero, el análisis estadístico de datos a partir de la frecuencia y los valores porcentuales empleando el programa informático SPSS versión 26; y, en un segundo momento, el examen interpretativo de los resultados a través de un análisis de la situación observada.

\section{Resultados}

La investigación plantea el tratamiento de los resultados considerando cuatro criterios de análisis: 1) los hábitos de lectura de los universitarios; 2) las preferencias de lectura de los estudiantes; 3 ) los efectos de la lectura en el desempeño académico de los jóvenes; y 4) las opiniones negativas sobre la lectura. En tal sentido, el cuestionario de 40 ítems se ha dividido para su análisis en cuatro tablas. 
Tabla 1

Hábitos de lectura en los universitarios

\begin{tabular}{|c|c|c|c|c|}
\hline \multirow[t]{2}{*}{ Ítems } & \multicolumn{2}{|c|}{ Sí, es cierto } & \multicolumn{2}{|c|}{$\begin{array}{c}\text { No, no es } \\
\text { verdad (que) }\end{array}$} \\
\hline & $\mathrm{F}$ & $\%$ & $\mathrm{~F}$ & $\%$ \\
\hline Me gusta leer en mi tiempo libre. & 37 & 69 & 17 & 31 \\
\hline $\begin{array}{l}\text { Me gusta tomar prestados libros de la biblioteca para } \\
\text { leer. }\end{array}$ & 28 & 52 & 26 & 48 \\
\hline $\begin{array}{l}\text { Me gusta leer solo los más vendidos (bestsellers) o los } \\
\text { recomendados. }\end{array}$ & 23 & 43 & 31 & 57 \\
\hline $\begin{array}{l}\text { Las características formales de un libro (grosor, tamaño } \\
\text { de fuente, ilustraciones, etc.) afectan mi preferencia por } \\
\text { leerlo o no. }\end{array}$ & 36 & 67 & 18 & 33 \\
\hline $\begin{array}{l}\text { Normalmente leo el libro en versión electrónica }(e- \\
\text { book). }\end{array}$ & 28 & 52 & 26 & 48 \\
\hline $\begin{array}{l}\text { Normalmente encuentro el libro que leeré en la } \\
\text { biblioteca. }\end{array}$ & 21 & 39 & 33 & 61 \\
\hline Generalmente suelo comprar el libro que leeré. & 24 & 44 & 30 & 56 \\
\hline $\begin{array}{l}\text { Inicialmente aprendo sobre el libro que leeré si mis } \\
\text { amigos lo leen. }\end{array}$ & 20 & 37 & 34 & 63 \\
\hline Creo que la lectura es una parte indispensable de la vida. & 43 & 80 & 11 & 20 \\
\hline Leo un texto en Internet debido a su bajo costo. & 41 & 76 & 13 & 24 \\
\hline
\end{tabular}

En la tabla 1 se muestran los hallazgos sobre el hábito lector en los estudiantes. Se observa claramente que, de todos los jóvenes universitarios, solo al $69 \%$ de ellos les gusta leer en su tiempo libre; pese a que el $80 \%$ de los estudiantes considera a la lectura una actividad indispensable en la vida. Además, en relación con los libros que leen los estudiantes, a la mitad de ellos o el $52 \%$ de los universitarios les gusta leer los libros de la biblioteca, a pesar de que el $61 \%$ no encuentra los libros que desea en este lugar. Por tal motivo, la mayoría de los jóvenes (el
76\%) optan como alternativa, la lectura de textos en Internet por su fácil accesibilidad y bajo costo; lo cual se refuerza con el hecho de que la mitad de los universitarios (el $52 \%$ les agrada leer versiones digitalizadas (libros electrónicos). Por otro lado, cerca de la mitad de los estudiantes (el $43 \%$ ) prefieren leer las obras recomendadas por sus profesores, antes que los libros que están leyendo sus compañeros o que fueron sugeridos por sus amigos (el 37\%). Y esta misma proporción de universitarios (el 44\%) comprará los libros para leer, los cuales en un $67 \%$ 
tendrán muy en cuenta al momento de la adquisición, las características formales del libro (grosor, tamaño de fuente, ilustraciones, etc.).

Tabla 2

Preferencias de lectura de los estudiantes universitarios

\begin{tabular}{lcccc}
\hline \multicolumn{1}{c}{ Ítems } & \multicolumn{2}{c}{ Sí, es cierto } & \multicolumn{2}{c}{ No, no es } \\
& & & \multicolumn{2}{c}{ verdad (que) } \\
\cline { 2 - 6 } & $\mathrm{F}$ & $\%$ & $\mathrm{~F}$ & $\%$ \\
\hline Me gusta leer obras literarias de autores peruanos. & 36 & 67 & 18 & 33 \\
Me gusta leer obras literarias de autores extranjeros. & 52 & 96 & 02 & 4 \\
Me gusta leer tesis de grado e informes de investigación. & 29 & 54 & 25 & 46 \\
Me gusta leer manuales académicos o libros científicos. & 30 & 56 & 24 & 44 \\
Me gusta leer periódicos. & 45 & 83 & 09 & 17 \\
Me gusta leer textos en línea. & 41 & 76 & 13 & 24 \\
Leo artículos académicos porque aprendo sobre nuevos & 53 & 98 & 01 & 2 \\
estudios en mi campo. & & & & \\
Sigo al menos una revista de cultura, arte y/o literatura de & 18 & 33 & 36 & 67 \\
forma semanal o mensualmente. & & & & \\
Prefiero ver una película de un libro literario en lugar de & 34 & 63 & 20 & 37 \\
leerlo. & & & & 10 \\
Mi texto literario favorito es la novela o el cuento. & 44 & 81 & 10 & 19 \\
Mi texto literario favorito es la poesía. & 12 & 22 & 42 & 78 \\
Me gusta leer textos dramáticos. & 20 & 37 & 34 & 63 \\
Me gusta leer libros sobre todos los temas. & 24 & 44 & 30 & 56 \\
\hline
\end{tabular}

En la tabla 2 se observa las preferencias de lectura de los estudiantes. Los resultados evidencian con un $96 \%$ que la gran mayoría de jóvenes prefieren leer libros de autores extranjeros a libros nacionales, pues la preferencia por autores peruanos no superó el $67 \%$. Asimismo, con un resultado de $98 \%$ se observó que a casi todos los estudiantes les gusta leer artículos académicos porque aprenden algo nuevo de su campo profesional, superando el 54\% que representan las consultas de tesis de grado o informes de investigación $\mathrm{y}$ superando el 56\% la lectura de manuales académicos o libros científicos. Igualmente, se muestra mucho interés en la lectura de textos recreativos como los periódicos con un $83 \%$ y las novelas o cuentos con un $81 \%$. Asimismo, existe una proporción alta de $76 \%$ de lectura de textos en línea a través de Internet. Contrariamente, los resultados revelan que el $67 \%$ de universitarios, generalmente, no siguen de forma recurrente ningún tipo de revista cultural, de arte, literatura. En ese mismo sentido, en relación a las obras literarias, el $60 \%$ de los estudiantes prefiere ver películas de un libro literario en lugar de leerlo, lo cual evidencia una preferencia por los textos audiovisuales o videográficos respecto de 
los textos escritos. Finalmente, con un 56\% se determina que más de la mitad de los estudiantes universitarios no tiene el interés por leer temas distintos a los de su formación profesional que involucren otros campos del saber.

\section{Tabla 3}

Efectos de la lectura en el desempeño de los estudiantes

\begin{tabular}{lccccc}
\hline \multicolumn{1}{c}{ Ítems } & \multicolumn{2}{c}{ Sí, es cierto } & \multicolumn{2}{c}{$\begin{array}{c}\text { No, no es } \\
\text { verdad (que) }\end{array}$} \\
\cline { 2 - 6 } & & $\mathrm{F}$ & $\%$ & $\mathrm{~F}$ & $\%$ \\
\hline $\begin{array}{l}\text { Leo para aprender. } \\
\text { Leo por placer. }\end{array}$ & 52 & 96 & 02 & 4 \\
La lectura nos hace adquirir el hábito de razonar & 39 & 91 & 05 & 9 \\
rápidamente. & & & 16 & 30 \\
La lectura es un canal para adquirir conocimiento del & 42 & 78 & 12 & 22 \\
mundo real. & & & & \\
Creo que la lectura influye positivamente en mi éxito & 52 & 96 & 02 & 4 \\
durante los exámenes. & 40 & 74 & 14 & 26 \\
La lectura me permite expresar mis sentimientos. & 50 & 93 & 04 & 7 \\
La lectura desarrolla el razonamiento múltiple. & 54 & 100 & 00 & 0 \\
La lectura mejora nuestra capacidad mental. & 52 & 96 & 02 & 4 \\
La lectura desarrolla nuestro mundo imaginario. & 50 & 93 & 04 & 7 \\
La lectura abre la puerta al mundo desconocido. & & & & \\
\hline
\end{tabular}

En la tabla 3 se observa los efectos de la lectura en el rendimiento de los estudiantes. Así, en general, los estudiantes creen que la lectura puede ser eficaz en muchos aspectos de sus vidas. En este sentido, entre el 96 y 100\% de estudiantes universitarios manifiestan claramente que la lectura puede desarrollar su mundo imaginario y potenciar su capacidad mental respectivamente. Todo lo cual sustenta la importancia de la lectura para el desarrollo cognitivo en las funciones lógicas y las de creatividad. Además, entre el 91 y $93 \%$ de los universitarios también considera que la lectura puede dar placer estético, desarrollar el razonamiento múltiple $\mathrm{y}$ abrir la puerta al mundo desconocido. De otro lado, el $96 \%$ de los estudiantes leen para fomentar el aprendizaje y el éxito en las horas de exámenes, lo cual posibilita el éxito en el desempeño académico en la universidad. En menor medida, pero aún en proporción significativa los jóvenes universitarios en un $74 \%$ creen que la lectura les permite expresar sus sentimientos. 
Tabla 4

Opiniones negativas sobre la lectura

\begin{tabular}{lccccc}
\hline \multirow{1}{*}{\multicolumn{1}{c}{ Ítems }} & \multicolumn{2}{c}{$\begin{array}{c}\text { Sí, es } \\
\text { cierto }\end{array}$} & \multicolumn{2}{c}{$\begin{array}{c}\text { No, no es } \\
\text { verdad (que) }\end{array}$} \\
\cline { 2 - 6 } & $\mathrm{F}$ & $\%$ & $\mathrm{~F}$ & $\%$ \\
\hline No me gusta leer. & 08 & 15 & 46 & 85 \\
Encuentro la lectura como una actividad aburrida. & 16 & 30 & 38 & 70 \\
No tengo tiempo para leer. & 15 & 28 & 39 & 72 \\
Debido a mi gran carga de trabajo, no tengo tiempo libre & 30 & 56 & 24 & 44 \\
para leer sobre ningún otro tema distinto a los de mis & & & & \\
cursos. & & & & \\
No leo porque creo que los libros son demasiado caros. & 13 & 24 & 41 & 76 \\
No leo porque soy de la opinión de que leer es una pérdida & 07 & 13 & 47 & 87 \\
de tiempo. & & & & \\
Encuentro la lectura como una actividad innecesaria. & 06 & 11 & 48 & 89 \\
\hline
\end{tabular}

En la tabla 4 se observan los resultados acerca de las opiniones negativas de los estudiantes sobre la lectura. A medida que se examinan las opiniones negativas de los estudiantes sobre la lectura, se muestra claramente que entre el 11 y $15 \%$ de los estudiantes no les gusta leer y consideran que la lectura es una actividad innecesaria y una pérdida de tiempo, lo cual demuestra que el hábito de lectura está desarrollado en la mayoría de los universitarios pues tienen una actitud general favorable hacia la lectura. Además, el 28 y 30\% afirma que la lectura es una actividad aburrida y no tiene tiempo para leer, lo cual se sustenta con el resultado de que una mayoría de estudiantes que representa el $56 \%$ no leen otros temas distintos a los de sus cursos, debido a que no tienen tiempo libre y por la gran carga de trabajo que supone ser estudiante universitario. Finalmente, la condición anterior se complementa por el hecho de que el $24 \%$ de universitarios no leen debido a que consideran que los libros son demasiado caros.

\section{Discusión}

A partir de los hallazgos generales observados en el estudio, se evidencia que la mayoría de estudiantes de universidades públicas peruanas tienen un buen hábito de lectura y una actitud positiva hacia las actividades lectoras. Sin embargo, hay un grupo minoritario de estudiantes (cantidad que no deja de ser significativa) en los que el hábito de lectura se muestra muy débil, puesto que en ellos la lectura se encuentra condicionada a la necesidad académica. Por tanto, la ausencia del interés personal o el apego cotidiano por leer no han permitido que se constituya el hábito lector. A pesar de lo anterior, un aspecto favorable es que la información del estudio demuestra que a más de la mitad de los estudiantes les agrada leer en el tiempo libre $\mathrm{y}$ lo 
consideran parte indispensable de sus vidas.

Por otro lado, respecto a la actitud general hacia lectura, se observa que los estudiantes son bastante selectivos, ya que toman en cuenta las características formales de los libros antes de leerlos y no están influenciados por las preferencias de los demás, por ejemplo, sus amigos. No obstante ello, manifiestan tener problemas para encontrar libros de su interés en la biblioteca universitaria, debido a que, generalmente, no existe un banco suficiente de libros; aunque sí se pueden encontrar otras fuentes similares. Por consiguiente, a veces leen los libros en versión electrónica (e-books) y con frecuencia leen textos de Internet o de soportes digitales de lectura en lugar de comprarlos. Dichos hallazgos son similares a estudios anteriores realizados por Lourdes de la Puente (2015) y Giovanni Parodi et al. (2019). En las investigaciones anteriores se demuestra que los jóvenes universitarios prefieren los medios electrónicos y los materiales de Internet. Este es un fenómeno que se ha popularizado debido a que los estudiantes pueden acceder a los textos deseados desde Internet; pues es mucho más fácil y simple que buscarlos en la biblioteca (Federación de Gremios de Editores de España, 2019).

En relación con la preferencia de lectura de los universitarios, a los estudiantes de universidades públicas peruanas les gusta leer libros de autores extranjeros en lugar de autores peruanos. Asimismo, la preferencia de textos a leer se inclina por los libros disciplinares y los artículos académicos, los cuales son los más consultados porque a través de ellos se aprende algo nuevo sobre el campo de estudio (Parlindungan, Rahmatillah, \& Lisyati, 2021). Del mismo modo, se prefiere la lectura de diarios locales y obras de ficción narrativas como las novelas y los cuentos. Situación que se ha consolidado durante todo este periodo de confinamiento y restricciones debido a la pandemia de la Covid-19 en el Perú y el mundo (ERI-Lectura, 2020). Contrariamente, los estudiantes leen muy poco de poesía y teatro, y no siguen a ninguna revista (ausencia de suscripciones físicas o virtuales). A partir de los datos obtenidos, se puede inferir que los estudiantes tienden a leer temas que apoyan sus estudios o fines académicos en lugar de leer por placer.

Con respecto a los efectos de la lectura en el desempeño de los estudiantes, los hallazgos muestran que, en general, los estudiantes creen que la lectura puede ser efectiva en muchos aspectos de sus vidas. Creen que la lectura puede mejorar y desarrollar su capacidad mental y su mundo imaginario. Además, también consideran que la lectura puede brindarles placer estético, permitirles crear múltiples razonamientos y hacer comprensible lo desconocido en el mundo, con el objetivo de fomentar su propio aprendizaje $\mathrm{y}$ de prepararlos para las evaluaciones curriculares. En ese mismo sentido, los jóvenes universitarios creen que la lectura les posibilita expresar sentimientos; y que les desarrolla el hábito de razonar rápidamente. Por tanto, los hábitos de lectura de los estudiantes son beneficiosos para potenciar la capacidad individual, para expresar emociones y obtener placer (Petit, 2003). 
En cuanto a las opiniones negativas de los estudiantes hacia la lectura, según los datos encontrados muestran que la mayoría de ellos evidencia una respuesta positiva sobre los hábitos de lectura. No obstante, debido a la gran carga de trabajo de los universitarios, no tienen tiempo libre para leer ni tampoco pueden hacerlo porque consideran que los libros son demasiado caros. En fin, estos efectos y opiniones desde una perspectiva cualitativa en la investigación educativa sobre gustos e intereses de lectura permiten caracterizar el fenómeno y establecer criterios o dimensiones de las variables involucradas en el estudio: preferencias lectoras $\mathrm{y}$ hábitos lectores (Loayza, 2006; LoayzaMaturrano, 2021 b).

De otro lado, aun cuando existen limitaciones respecto del muestreo por ser no probabilístico, las condiciones que se establecieron en la ejecución del instrumento permiten de forma suficiente recoger la información necesaria para determinar las características y revalidar los criterios propuestos en el cuestionario de preferencias y hábitos de lectura. Sin embargo, vistos los resultados y expresada la discusión de los mismos se posibilita la realización de estudios posteriores que vinculen en el hábito lector no solo la actitud general favorable hacia la lectura, sino también los efectos y percepciones negativas al respecto, para ponderar los niveles de formación del hábito de lectura en los sujetos. Así, la investigación resalta la importancia de los hábitos de lectura en los estudiantes universitarios a través de la vinculación de las actitudes generales hacia la lectura y las opiniones negativas sobre ésta, así como la relación existente entre los gustos lectores y los efectos de la lectura. Todo lo cual establece repercusiones teóricas-metodológicas en el abordaje del estudio sobre la lectura.

\section{Conclusiones}

Una primera conclusión retomando los estudios previos de Ögeyik \& Akyay, 2009; García-Delgado, 2011; Travieso et al, 2013; De la Puente, 2015; Jiménez-Pérez, 2018; Parodi, et al. 2019, es que se puede afirmar que las preferencias de lectura y el hábito de lectura de los estudiantes de universidades públicas peruanas son ciertamente similares a los de los estudiantes de otras latitudes (Turquía, España, Chile). No obstante, hay diferencias en cuando a la mayor o menor posibilidad económica para la adquisición de libros y el ratio anual de tasa de lectoría por estudiante respecto a estudiantes ingleses y españoles (Gleed, 2013; Fraga, 2019); así como en el caso de los universitarios ingleses y españoles que suelen suscribirse a algunas revistas con el objetivo de seguirlas.

Una segunda conclusión es que los estudiantes universitarios tienen hábitos lectores y preferencias de lectura, porque según los datos obtenidos se demuestra que son lectores conscientes: investigan sobre los libros antes de comprarlos y por la tendencia general a elegir sus textos siempre que necesiten información sobre un tema que les interesa. Asimismo, pese a que el presente estudio parte de una iniciativa de carácter académico y constituye simplemente una aproximación estadística descriptiva y reflexiva sobre las preferencias de lectura y los hábitos 
lectores de los estudiantes universitarios de una zona urbana de Lima, los datos, el análisis de los mismos y las conclusiones pueden ser útiles para la implementación de estrategias y políticas educativas de

\section{Referencias}

Álvarez, Y. y Fabregat, S. (2017). Representaciones, hábitos y dificultades de composición del texto escrito en estudiantes universitarios: un estudio exploratorio. Investigaciones sobre lectura, (8), 60-78. https://www.comprensionlectora. es/revistaisl/index.php/revistaISL /article/view/217

Bao, X. (2019). Critical thinking and critical reading: A sound pedagogical paring International. Journal of Continuing Engineering Education and Life-Long Learning, 29(1-2), 129-147.

https://doi.org/10.1504/IJCEELL.2 019.099258

Casiraghi, B., \& Soares, J. (2021). Avaliação do Ensino Superior brasileiro: Desempenho dos estudantes em formação geral. Revista Portuguesa de Educação, 34(1). https://doi.org/10.21814/rpe.208 21

De la Puente, L. (2015). Motivación hacia la lectura, hábito de lectura $y$ comprensión de textos en estudiantes de psicología de dos universidades particulares de Lima (Tesis de maestría, Universidad Peruana Cayetano Heredia, Lima - Perú]. fomento de la lectura en los claustros universitarios por parte de órganos directivos de una universidad o inclusive de todo un sistema universitario.

https://core.ac.uk/download/pdf/ 143615059.pdf

Delgado, C. (2020). La crisis covid-19 y el cambio educativo. Revista CIEG del Centro de Investigación y Estudios Gerenciales, 46, 175-194. http://revista.grupocieg.org/public acion/revista-cieg-n-46noviembre-diciembre-2020/

Domínguez, I., Rodríguez, L., Torres, Y. y Ruiz, M. (2015). Importancia de la lectura y la formación del hábito de leer en la formación inicial. Estudios del Desarrollo Social: Cuba y América Latina, 3(1), 94-102. http://www.revflacso.uh.cu/index. php/EDS/article/view/80/80

Elche, M. y Yubero, S. (2019). La influencia del hábito lector en el empleo de internet: un estudio con jóvenes universitarios. Investigación Bibliotecológica, 33(79), 51-66. http://dx.doi.org/10.22201/iibi.24 488321xe.2019.79.57985

ERI-Lectura. (2020). Hábitos lectores durante el confinamiento por la covid-19 [Informe ejecutivo]. RODERIC (Universitat de València). http://roderic.uv.es/ 
Federación de Gremios de Editores de España (2019). Hábitos de Lectura y Compra de libros en España 2018. Madrid, Conecta. https://www.federacioneditores.or g/lectura-y-compra-de-libros2018.pdf

Flores, D. (2016). La importancia del hábito de la lectura, reacción y pensamiento. Revista del Instituto de Estudios en Educación Universidad del Norte, (24), 128135.

http://www.scielo.org.co/scielo.ph p?pid=S2145-

94442016000100010\&script=sci_a bstract\&tlng=es

Fraga, J. (2019). En busca del lector: una mirada a los hábitos de lectura en España (Trabajo de grado). Facultad de Sociología, Universidad La Coruña.

http://hdl.handle.net/2183/23608

García-Delgado, B. (2011). Estudio de los hábitos lectores de los estudiantes de la Universidad Europea de Madrid. Ibersid. Revista de sistemas de información y documentación, 5, 99-107.

https://ibersid.eu/ojs/index.php/i bersid/article/view/3890

Gleed, A. (2013). Booktrust Reading Habits Survey 2013: A national survey of reading habits and attitudes to books amongst adults in England. London, DJS Research Limited. https://www.booktrust.org.uk/glo balassets/resources/research/157 6-booktrust-reading-habits-reportfinal.pdf

Guevara, M. (2017). Adquisición de hábitos de lectura y escritura [Tesis de magister, Universidad Pontifica Bolivariana]. https://repository.upb.edu.co/han dle $/ 20.500 .11912 / 3375$ ?show=full

Jiménez-Pérez, E. (2018). La inteligencia emocional como predictor del hábito de lectura y la competencia lectora en estudiantes universitarios. Investigaciones sobre Lectura. Málaga, 10, 30-54. https://doi.org/10.37132/isl.v0i10 .256

Kaya, A. Í., Kaya, A., \& Bindak, R. (2020). An Investigation Of Teachers' Attitudes And Behaviors Towards Reading Books During The Covid-19 Pandemic. Milli Egitim, 49(1), 737755. https://doi.org/10.37669/milliegit im.785990

Loayza, E. (2006). Investigación cualitativa en Educación. Investigación educativa, 10(18), 75-85. https://revistasinvestigacion.unms m.edu.pe/index.php/educa/article /view/3778

Loayza, E. (2020). La investigación cualitativa en Ciencias Humanas y Educación. Criterios para elaborar artículos científicos. Educare et Comunicare, 8(2), 56-66. https://doi.org/10.35383/educare. v8i2.536 
Loayza-Maturrano, E. (2021 a). Enfoques modernos para determinar el nuevo rol del docente. Sciéndo, 24(3), 177183.

https://doi.org/10.17268/sciendo. 2021.023

Loayza-Maturrano, E. $\quad(2021 \quad$ b). $\quad$ El arborigrama. Estrategia didáctica de comprensión lectora de textos narrativos. Investigación Valdizana, 15(2), 89-100. https://doi.org/10.33554/riv.15.2. 1005

Magadán-Díaz, M. \& Rivas-García, J. (2019). The impact of digitization in Spanish scholarly publishers. LIBER Quarterly, 29(1), 1-22. http://doi.org/10.18352/lq.10261

Muñoz, C., Valenzuela, J., Avendaño, C. y Nuñez, C. (2016). Mejora en la motivación por la lectura académica: la mirada de estudiantes motivados. Ocnos, Revista de estudios sobre lectura, 15, (1), 52-68. https://doi.org/10.18239/ocnos_2 016.15.1.941

Ögeyik, M. \& Akyay, E. (2009). Investigating Reading Habits and Preferences of Student Teachers at Foreign Language Departments. The International Journal of Language Society and Culture. 8, 72-80. https://aaref.com.au/wpcontent/uploads/2018/05/287.pdf

Parodi, G., Moreno-de-León, T., Julio, C. y Burdiles, G. (2019). Generación Google o generación Gutenberg: hábitos y propósitos de lectura en estudiantes universitarios chilenos. Comunicar, 27(58), 85-94. https://doi.org/10.3916/C582019-08

Parlindungan, F., Rahmatillah, R., \& Lisyati, L. (2021). Academic Reading Preferences and Behaviors of Indonesian Undergraduate Students during Covid-19 Pandemic. TESOL International Journal, 16(4.1), 6-27. http://conference.ucyp.edu.my/wp

content/uploads/2020/11/Exampl e-of-Abstract-1.pdf

Petit, M. (2003). La lectura íntima y compartida. (Ponencia). I Jornadas Aragonesas de bibliotecas escolares y promoción de la lectura. Décimo aniversario: Leer Juntos. Ballobar. https://docer.com.ar/doc/nc1nnx

Salazar, S., y Ponce, D. (1999). Hábitos de lectura (1ra. ed.). Libro y la lectura.

Salvador, J. y Agustín, M. (2015). Hábitos de lectura y consumo de información en estudiantes de la Facultad de Filosofía y Letras de la Universidad de Zaragoza. Anales de Documentación, (18), 1-4 http://dx.doi.org/10.6018/analesd oc.18.1.201971

Sotelo, R. y Espíritu, J. (2020). Formación general humanista en universidades públicas. Revista Ciencias y Humanidades, 10(10), 277-95.

https://revistacienciasyhumanidad 
es.com/index.php/home/article/vi ew/113

Travieso, M., Méndez, B. y Ineraite, R. (2013). La lectura y los hábitos lectores. Consideraciones teóricometodológicas. Revista Conrado, $9(40)$, 62-68. https://conrado.ucf.edu.cu/index.p $\mathrm{hp} /$ conrado/article/view/95. 